INTERNATIONAL JOURNAL
OHF
PHARMACEUTICAL SCIENCES
RESEARCH

Received on 19 August, 2012; received in revised form 15 September, 2012; accepted 25 November, 2012

\title{
FORMULATION AND EVALUATION OF MOUTH DISSOLVING TABLET
}

Vishal Sahu* and B.V. Bakade

P. Wadhwani College of Pharmacy, Amravti University, Amravti, Maharashtra, India

Keywords:

Rapid disintegration, Superdisintegrant,

Venlafaxine, In vitro Dispersion Time

Correspondence to Author:

Vishal Sahu

P. Wadhwani College of Pharmacy, Amravti University, Amravti,

Maharashtra, India

E-mail: vishal1002feb@gmail.com

\begin{tabular}{|c|c|}
\hline QUICK RESPONSE CODE & IJPSR: \\
\hline IE & ICV (2011)- 5.07 \\
& Website: \\
\hline
\end{tabular}

\begin{abstract}
The objective of the present study was to develop venlafaxine hydrochloride orodispersible tablet by using conventional techniques which are simple and cost effective such as use of superdisintegrant technology. In this, sodium starch glycolate and Crosscarmellose sodium crosspovidone and kyron-T-314 were used in the rapid disintegration of the tablets. In this various trials were conducted for the selection of optimum concentration of superdisintegrant. The optimized formula aids in the stabilization of final product. The blend and compressed tablets were evaluated for physical characteristics like bulk density, tapped density, angle of repose, hardness, friability, disintegration time, wetting time, water absorption ratio, In-vitro dispersion time and chemical characteristics like In-vitro dissolution, content uniformity and assay. The stability study was conducted for the optimized batch. This design of dosage form will open a new era for rapid disintegration tablets.
\end{abstract}

INTRODUCTION: Mouth dissolving drug delivery systems (MDDDS) are a new generation of formulations which combine the advantages of both liquid and conventional tablet formulations, and at the same time, offer added advantages over both the traditional dosage forms. It provides the convenience of a tablet formulation and allows the ease of swallowing provided by a liquid formulation. The formulations have special advantages for dysphasic, geriatric, pediatric, bed-ridden, travelling and psychotic patients who are unable to swallow or refuse to swallow conventional oral formulations. It do not require water for administration, thus are good alternative for travellers and for bed ridden patients ${ }^{1}$.

In the recent past, several new advanced technologies have been introduced for the formulation of mouth dissolving tablets (MDTs) with very interesting features, like extremely low disintegration time, exceptional taste masking ability, pleasant mouth feel and sugar free tablets for diabetic patients. The technologies utilized for fabrication of MDDDS include lyophilization, moulding, direct compression, cotton candy process, spray drying, sublimation, mass extrusion, and quick dissolve film formation. These techniques are based on the principles of increasing porosity and/or addition of super disintegrants and water soluble excipients in the tablets.

The formulations prepared from these techniques differ from each other on the basis of the factors like mechanical strength of final product, drug and dosage form stability, mouth feel, taste, rate of dissolution of the formulation in saliva, rate of absorption from saliva and overall drug bioavailability. These products not only increase the patient"s compliance but also fetch large revenues to manufacturers due to line extension of the existing formulation ${ }^{2}$. Although, numerous technologies had been developed for the fabrication of these unique dosage forms in last two decades, but so 
far, no standardized technique has been designed or mentioned in pharmacopoeias for their evaluation except in European Pharmacopoeia (EP), which defines orodispersible tablets as "uncoated tablets intended to be placed in the mouth where they disperse rapidly before being swallowed". European Pharmacopoeia also specifies that the orodispersible tablets should disintegrate within 3 minutes when subjected to conventional disintegration test used for tablets and capsules ${ }^{3}$.

\section{MATERIALS AND METHODS:}

Materials: Venlafaxine Hydrochloride was obtained from Orchid Chemicals and Pharmaceuticals Ltd., Sodium starch glycollate and Crosscarmellose sodium were obtained from Rankem limited, Mumbai. Other excipients used in this formulation were of analytical grade.
Methods:

Preparation of Venlafaxine Orodispersible tablets: Venlafaxine Hydrochloride, Microcrystalline cellulose, Superdisintegrants, Aerosil, Aspartame, Mannitol, Starch-1500 were sifted through \# 40 mesh separately, collected in poly bags. Venlafaxine hydrochloride, Microcrystalline cellulose, Super- disintegrants, Aerosil, Aspartame, Mannitol, Starch-1500, Strawberry flavor were loaded into Octagonal blender and mixed. Sodium saccharine, Magnesium stearate, Talc were added to this and mixed for 10 minutes, then sifted through \#60 mesh.

Then, the final blend was compressed in to tablets using Rotary press tablet compression machine. The formula for the preparation of various baches of Venlafaxine hydrochloride tablets were given in table 1 and 2.

TABLE 1: COMPOSITION OF ALL THE FORMULATIONS (MD1-MD8)

\begin{tabular}{cccccccccc}
\hline Sr. No & Ingredient (mg/tablet) & MD 1 & MD 2 & MD 3 & MD 4 & MD 5 & MD 6 & MD 7 & MD 8 \\
\hline $\mathbf{1}$ & Venlafaxine & 25 & 25 & 25 & 25 & 25 & 25 & 25 & 25 \\
$\mathbf{2}$ & Crosscarmellose sodium & 5 & 10 & 15 & 20 & - & - & - & - \\
$\mathbf{3}$ & Sodium starch glycolate & - & - & - & - & 5 & 10 & 15 & 20 \\
$\mathbf{4}$ & Avicel & 46 & 46 & 46 & 46 & 46 & 46 & 46 & 46 \\
$\mathbf{5}$ & Mannitol & 60 & 55 & 50 & 45 & 60 & 55 & 50 & 45 \\
$\mathbf{6}$ & Talc & 4 & 4 & 4 & 4 & 4 & 4 & 4 & 4 \\
$\mathbf{7}$ & Aspartame & 18 & 18 & 18 & 18 & 18 & 18 & 18 & 18 \\
$\mathbf{8}$ & Aerosil & 5 & 5 & 5 & 5 & 5 & 5 & 5 & 5 \\
$\mathbf{9}$ & Magnesium stearate & 2 & 2 & 2 & 2 & 2 & 2 & 2 & 2 \\
$\mathbf{1 0}$ & Mint flavor & 5 & 5 & 5 & 5 & 5 & 5 & 5 & 5 \\
$\mathbf{1 1}$ & Total weight & 170 & 170 & 170 & 170 & 170 & 170 & 170 & 170 \\
\hline
\end{tabular}

TABLE 2: COMPOSITION OF ALL THE FORMULATIONS (MD9-MD16)

\begin{tabular}{cccccccccc}
\hline Sr. No & Ingredient (mg/tablet) & MD 9 & MD 10 & MD 11 & MD 12 & MD 13 & MD 14 & MD 15 & MD 16 \\
\hline $\mathbf{1}$ & Venlafaxine & 25 & 25 & 25 & 25 & 25 & 25 & 25 & 25 \\
$\mathbf{2}$ & Kyron T-314 & 5 & 10 & 15 & 20 & - & - & - & - \\
$\mathbf{3}$ & L-HPC & - & - & - & - & 5 & 10 & 15 & 20 \\
$\mathbf{4}$ & Avicel & 46 & 46 & 46 & 46 & 46 & 46 & 46 & 46 \\
$\mathbf{5}$ & Mannitol & 60 & 55 & 50 & 45 & 60 & 55 & 50 & 45 \\
$\mathbf{6}$ & Talc & 4 & 4 & 4 & 4 & 4 & 4 & 4 & 4 \\
$\mathbf{7}$ & Aspartame & 18 & 18 & 18 & 18 & 18 & 18 & 18 & 18 \\
$\mathbf{8}$ & Aerosil & 5 & 5 & 5 & 5 & 5 & 5 & 5 & 5 \\
$\mathbf{9}$ & Magnesium stearate & 2 & 2 & 2 & 2 & 2 & 2 & 2 & 2 \\
$\mathbf{1 0}$ & Mint flavor & 5 & 5 & 5 & 5 & 5 & 5 & 5 & 5 \\
$\mathbf{1 1}$ & Total weight & 170 & 170 & 170 & 170 & 170 & 170 & 170 & 170 \\
\hline
\end{tabular}

\section{Evaluation:}

Pre-compression parameters: The Angle of repose, Bulk density, Tapped density, Compressibility Index, Hausner's ratio and \% LOD were determined and results were given in Table 3.
Post compression parameters: Thickness, Weight Variation Test, Hardness Test, Friability Test and Disintegration were determined as per the Standard Procedures and the results obtained are tabulated in Table 4 and 5 . 
TABLE 3: PREFORMULATION STUDIES OF DIFFERENT TABLET FORMULATION

\begin{tabular}{|c|c|c|c|c|c|}
\hline Formulation & Angle of repose $(\theta)$ & Bulk density (g/cc) & Tapped density (g/cc) & Carr's index (\%) & Hausner ratio \\
\hline F1 & $32.15 \pm 0.11$ & $0.293 \pm 0.16$ & $0.418 \pm 0.24$ & $29.90 \pm 0.14$ & $1.42 \pm 0.16$ \\
\hline $\mathrm{F} 2$ & $30.46 \pm 0.22$ & $0.308 \pm 0.35$ & $0.450 \pm 0.28$ & $31.55 \pm 0.18$ & $1.46 \pm 0.05$ \\
\hline F3 & $31.56 \pm 0.17$ & $0.304 \pm 0.18$ & $0.450 \pm 0.13$ & $32.44 \pm 0.25$ & $1.48 \pm 0.02$ \\
\hline $\mathrm{F} 4$ & $33.46 \pm 0.31$ & $0.296 \pm 0.24$ & $0.422 \pm 0.19$ & $31.42 \pm 0.33$ & $1.42 \pm 0.02$ \\
\hline F5 & $32.61 \pm 0.24$ & $0.318 \pm 0.27$ & $0.441 \pm 0.24$ & $29.89 \pm 0.37$ & $1.38 \pm 0.07$ \\
\hline F6 & $32.26 \pm 0.15$ & $0.328 \pm 0.34$ & $0.465 \pm 0.32$ & $29.46 \pm 0.28$ & $1.41 \pm 0.05$ \\
\hline F7 & $34.33 \pm 0.19$ & $0.316 \pm 0.25$ & $0.438 \pm 0.27$ & $27.85 \pm 0.26$ & $1.38 \pm 0.07$ \\
\hline F8 & $33.42 \pm 0.21$ & $0.366 \pm 0.26$ & $0.55 \pm 0.34$ & $33.4 \pm 0.34$ & $1.36 \pm 0.21$ \\
\hline F9 & $32.51 \pm 0.23$ & $0.309 \pm 0.41$ & $0.452 \pm 0.26$ & $31.63 \pm 0.21$ & $1.46 \pm 0.05$ \\
\hline F10 & $31.62 \pm 0.16$ & $0.309 \pm 0.31$ & $0.042 \pm 0.18$ & $26.42 \pm 0.16$ & $1.35 \pm 0.03$ \\
\hline F11 & $30.67 \pm 0.12$ & $0.322 \pm 0.24$ & $0.446 \pm 0.28$ & $27.80 \pm 0.21$ & $1.38 \pm 0.16$ \\
\hline F12 & $30.71 \pm 0.22$ & $0.307 \pm 0.15$ & $0.449 \pm 0.33$ & $31.62 \pm 0.23$ & $1.46 \pm 0.21$ \\
\hline F13 & $31.82 \pm 0.32$ & $0.328 \pm 0.21$ & $0.465 \pm 0.38$ & $29.46 \pm 0.30$ & $1.41 \pm 0.07$ \\
\hline F14 & $32.31 \pm 0.17$ & $0.316 \pm 0.19$ & $0.438 \pm 0.34$ & $24.85 \pm 0.27$ & $1.38 \pm 0.06$ \\
\hline F15 & $33.23 \pm 0.14$ & $0.318 \pm 0.23$ & $0.441 \pm 0.26$ & $29.89 \pm 0.26$ & $1.36 \pm 0.21$ \\
\hline F16 & $31.32 \pm 0.19$ & $0.366 \pm 0.21$ & $0.550 \pm 0.28$ & $33.45 \pm 033$ & $1.41 \pm 0.19$ \\
\hline
\end{tabular}

TABLE 4: EVALUATION RESULT OF TABLET FROM BATCH MD1-MD16

\begin{tabular}{ccccc}
\hline Formulation & Thickness $(\mathbf{c m})$ & Diameter $(\mathbf{c m})$ & Friability (\%) & Hardness (kg/cm $\left.{ }^{2}\right)$ \\
\hline MD1 & $0.384 \pm 0.14$ & $0.815 \pm 0.19$ & $0.60 \pm 0.15$ & $3.2 \pm 0.23$ \\
MD2 & $0.410 \pm 0.74$ & $0.815 \pm 0.13$ & $0.60 \pm 0.07$ & $3.1 \pm 0.14$ \\
MD3 & $0.410 \pm 0.21$ & $0.815 \pm 0.26$ & $0.29 \pm 0.05$ & $3.2 \pm 0.74$ \\
MD4 & $0.411 \pm 0.14$ & $0.815 \pm 0.33$ & $0.88 \pm 0.02$ & $3.3 \pm 0.47$ \\
MD5 & $0.409 \pm 0.32$ & $0.815 \pm 0.53$ & $0.60 \pm 0.06$ & $3.0 \pm 0.51$ \\
MD6 & $0.410 \pm 0.47$ & $0.815 \pm 0.11$ & $0.29 \pm 0.01$ & $3.2 \pm 0.54$ \\
MD7 & $0.409 \pm 0.54$ & $0.815 \pm 0.16$ & $0.60 \pm 0.08$ & $3.2 \pm 0.71$ \\
MD8 & $0.409 \pm 0.47$ & $0.815 \pm 0.22$ & $0.88 \pm 0.15$ & $3.3 \pm 0.62$ \\
MD9 & $0.410 \pm 0.47$ & $0.815 \pm 0.37$ & $0.60 \pm 0.17$ & $3.2 \pm 0.19$ \\
MD10 & $0.410 \pm 0.52$ & $0.815 \pm 0.41$ & $0.29 \pm 0.21$ & $3.2 \pm 0.27$ \\
MD11 & $0.409 \pm 0.51$ & $0.815 \pm 0.29$ & $0.29 \pm 0.03$ & $3.1 \pm 0.54$ \\
MD12 & $0.410 \pm 0.51$ & $0.815 \pm 0.17$ & $0.88 \pm 0.07$ & $3.1 \pm 0.36$ \\
MD13 & $0.409 \pm 0.48$ & $0.815 \pm 0.13$ & $0.29 \pm 0.11$ & $3.0 \pm 0.54$ \\
MD14 & $0.409 \pm 0.51$ & $0.815 \pm 0.14$ & $0.88 \pm 0.13$ & $3.1 \pm 0.49$ \\
MD15 & $0.410 \pm 0.46$ & $0.815 \pm 0.35$ & $0.60 \pm 0.27$ & $3.2 \pm 0.51$ \\
MD16 & $0.410 \pm 0.41$ & $0.815 \pm 0.43$ & $0.88 \pm 0.01$ & \\
\hline
\end{tabular}

TABLE 5: EVALUATION RESULT OF TABLET FROM BATCH MD1-MD16

\begin{tabular}{cccc}
\hline Formulation & Drug Content (\%) & Weight variation (mg) & Disintegration time (min) \\
\hline MD1 & $99.29 \pm 0.51$ & $171.3 \pm 0.12$ & $1.27 \pm 0.01$ \\
MD2 & $98.52 \pm 1.08$ & $171.6 \pm 0.53$ & $0.58 \pm 0.02$ \\
MD3 & $97.76 \pm 1.26$ & $169.8 \pm 0.54$ & $0.50 \pm 0.14$ \\
MD4 & $97.63 \pm 0.51$ & $170.3 \pm 0.63$ & $0.41 \pm 0.25$ \\
MD5 & $99.86 \pm 0.33$ & $172.5 \pm 0.87$ & $1.44 \pm 0.14$ \\
MD6 & $98.51 \pm 0.94$ & $171.8 \pm 0.36$ & $0.55 \pm 0.14$ \\
MD7 & $99.63 \pm 0.86$ & $171.1 \pm 0.74$ & $0.43 \pm 0.36$ \\
MD8 & $98.51 \pm 0.63$ & $172.2 \pm 0.52$ & $0.37 \pm 0.15$ \\
MD9 & $98.43 \pm 0.71$ & $171.1 \pm 0.14$ & $1.46 \pm 0.14$ \\
MD10 & $99.26 \pm 0.54$ & $173.3 \pm 0.51$ & $0.41 \pm 0.13$ \\
MD11 & $98.42 \pm 0.46$ & $170.6 \pm 0.49$ & $0.44 \pm 0.27$ \\
MD12 & $98.81 \pm 0.51$ & $170.8 \pm 0.37$ & $0.39 \pm 0.21$ \\
MD13 & $99.64 \pm 0.29$ & $169.9 \pm 0.68$ & $1.42 \pm 0.11$ \\
MD14 & $98.52 \pm 0.38$ & $173.5 \pm 0.73$ & $0.58 \pm 0.07$ \\
MD15 & $99.61 \pm 0.41$ & $172.8 \pm 0.81$ & $0.46 \pm 0.13$ \\
MD16 & $99.82 \pm 0.51$ & $172.1 \pm 0.72$ & $0.37 \pm 0.11$ \\
\hline
\end{tabular}


In vitro Drug Release: The tablets prepared from different batches are further evaluated for in-vitro drug release studies.

1. Cumulative Percent Drug Release of batch from MD1-MD4:

TABLE 6: PERCENT DRUG RELEASE OF TABLET FROM BATCH MD1-MD4

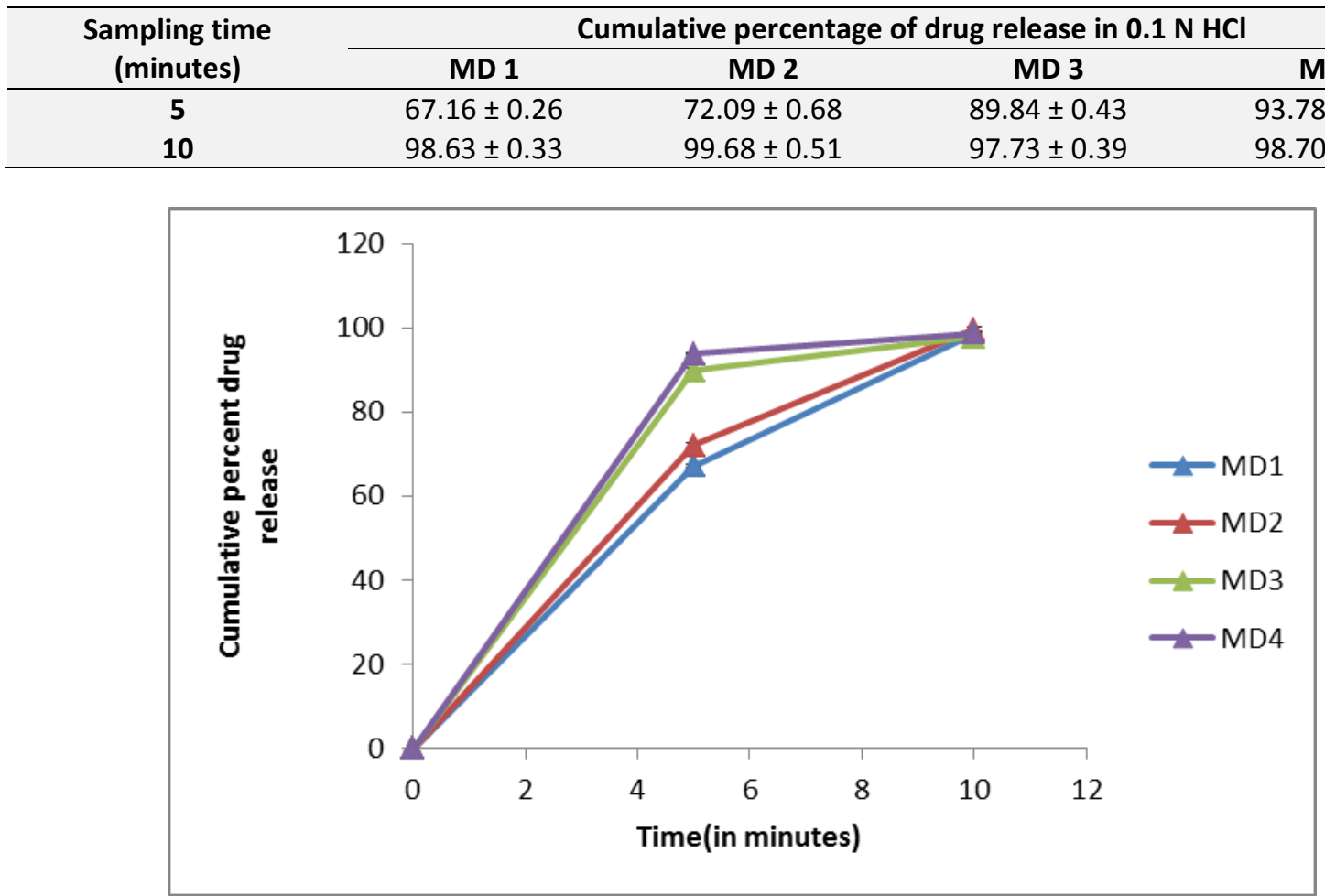

FIG. 1: COMPARISON OF CUMULATIVE PERCENT DRUG RELEASE FROM MD1 TO MD4

\section{Cumulative Percent Drug Release of batch from MD5-MD8:}

TABLE 7 : PERCENT DRUG RELEASE OF TABLET FROM BATCH MD5-MD8.

\begin{tabular}{ccccc}
\hline $\begin{array}{c}\text { Sampling time } \\
\text { (minutes) }\end{array}$ & \multicolumn{4}{c}{ Cumulative percentage of drug release in 0.1 N HCl } \\
\cline { 2 - 5 } & MD 5 & MD 6 & MD 7 & MD 8 \\
\hline $\mathbf{5}$ & $91.81 \pm 0.21$ & $92.80 \pm 0.31$ & $94.77 \pm 0.12$ & $95.75 \pm 0.53$ \\
$\mathbf{1 0}$ & $99.68 \pm 0.29$ & $97.73 \pm 0.33$ & $98.76 \pm 0.17$ & $96.75 \pm 0.66$ \\
\hline
\end{tabular}

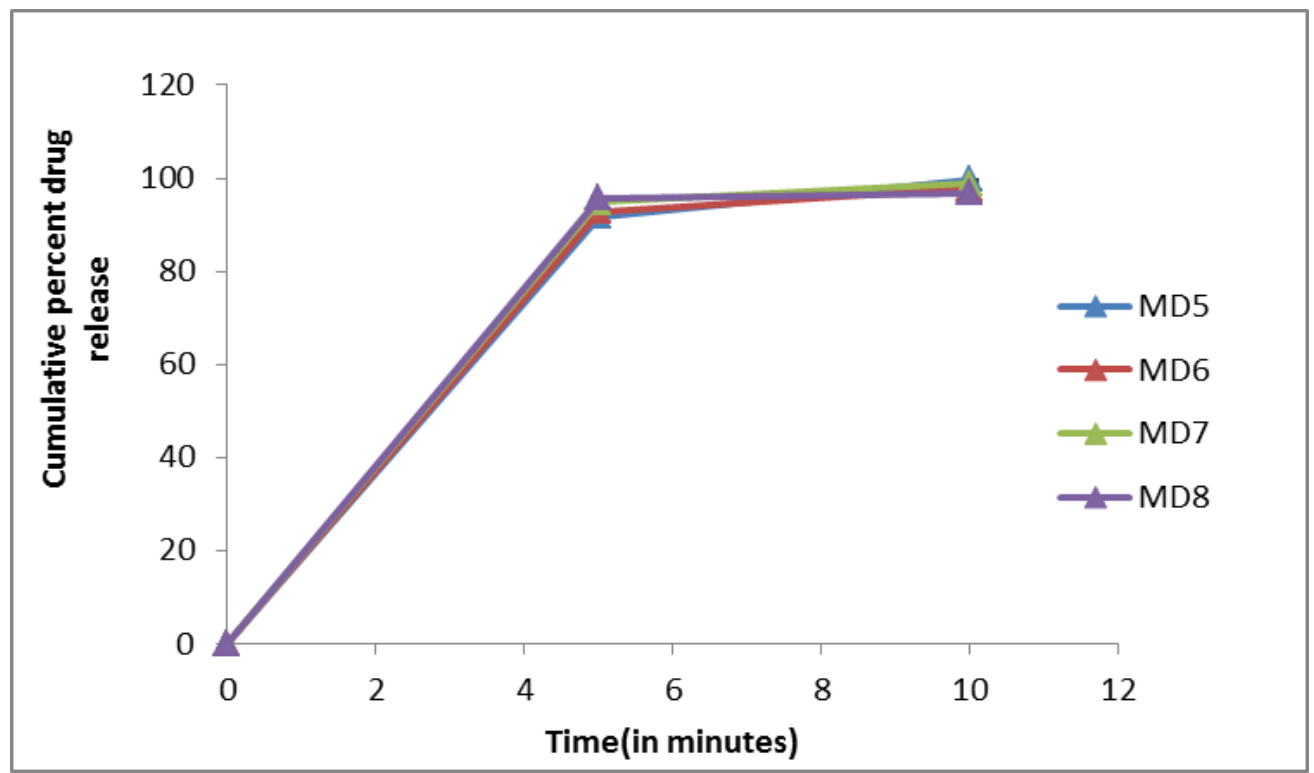

FIG. 2: COMPERISION OF CUMULATIVE PERCENT DRUG RELEASE FROM MD5 TO MD8 
3. Cumulative Percent Drug Release of batch from MD9-MD12:

TABLE 8: PERCENT DRUG RELEASE OF TABLET FROM BATCH MD9-MD12

\begin{tabular}{ccccc}
\hline $\begin{array}{c}\text { Sampling time } \\
\text { (minutes) }\end{array}$ & MD 9 & MD 10 & MD 11 & MD 12 \\
\hline $\mathbf{5}$ & $89.84 \pm 0.63$ & $88.85 \pm 0.47$ & $87.87 \pm 0.39$ & $91.81 \pm 0.19$ \\
10 & $97.73 \pm 0.59$ & $99.80 \pm 0.44$ & $97.87 \pm 0.21$ & $98.86 \pm 0.15$ \\
\hline
\end{tabular}

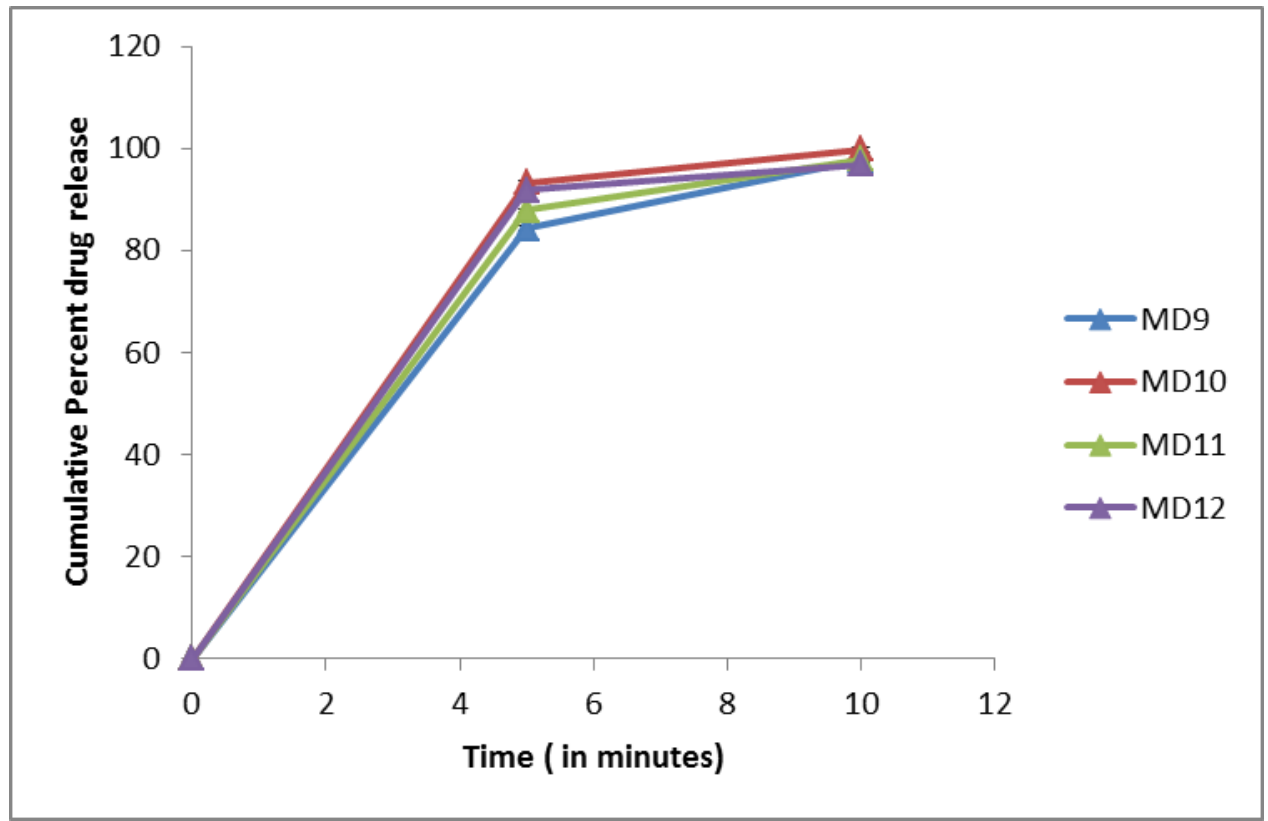

FIG. 3: COMPARISON OF CUMULATIVE PERCENT DRUG RELEASE FROM MD9 TO MD12

\section{Cumulative Percent Drug Release of batch from MD13-MD16:-}

TABLE 9 : PERCENT DRUG RELEASE OF TABLET FROM BATCH MD13-MD16.

\begin{tabular}{llccc}
\hline \multirow{2}{*}{$\begin{array}{c}\text { Sampling time } \\
\text { (minutes) }\end{array}$} & \multicolumn{4}{c}{ Cumulative percentage of drug release in 0.1 N HCl } \\
\cline { 2 - 5 } & MD 13 & MD 14 & MD 15 & MD 16 \\
\hline $\mathbf{5}$ & $89.84 \pm 0.88$ & $91.81 \pm 0.25$ & $88.85 \pm 0.78$ & $87.96 \pm 0.17$ \\
$\mathbf{1 0}$ & $99.68 \pm 0.76$ & $99.76 \pm 0.44$ & $98.70 \pm 0.45$ & $98.87 \pm 0.26$ \\
\hline
\end{tabular}

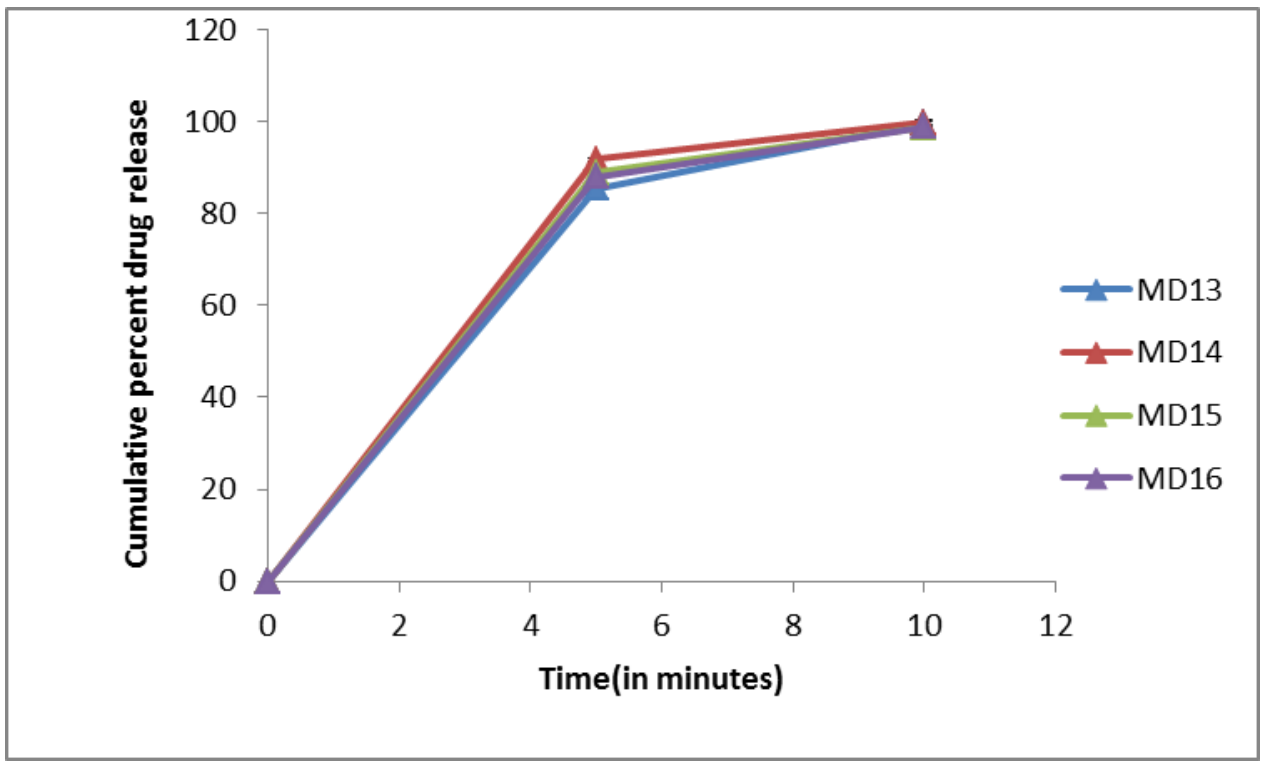

FIG. 4: COMPERISION OF CUMULATIVE PERCENT DRUG RELEASE FROM MD13 TO MD16 


\section{COMPATIBILITY STUDY:-}

\section{FTIR of Venlafaxine Hydrochloride}

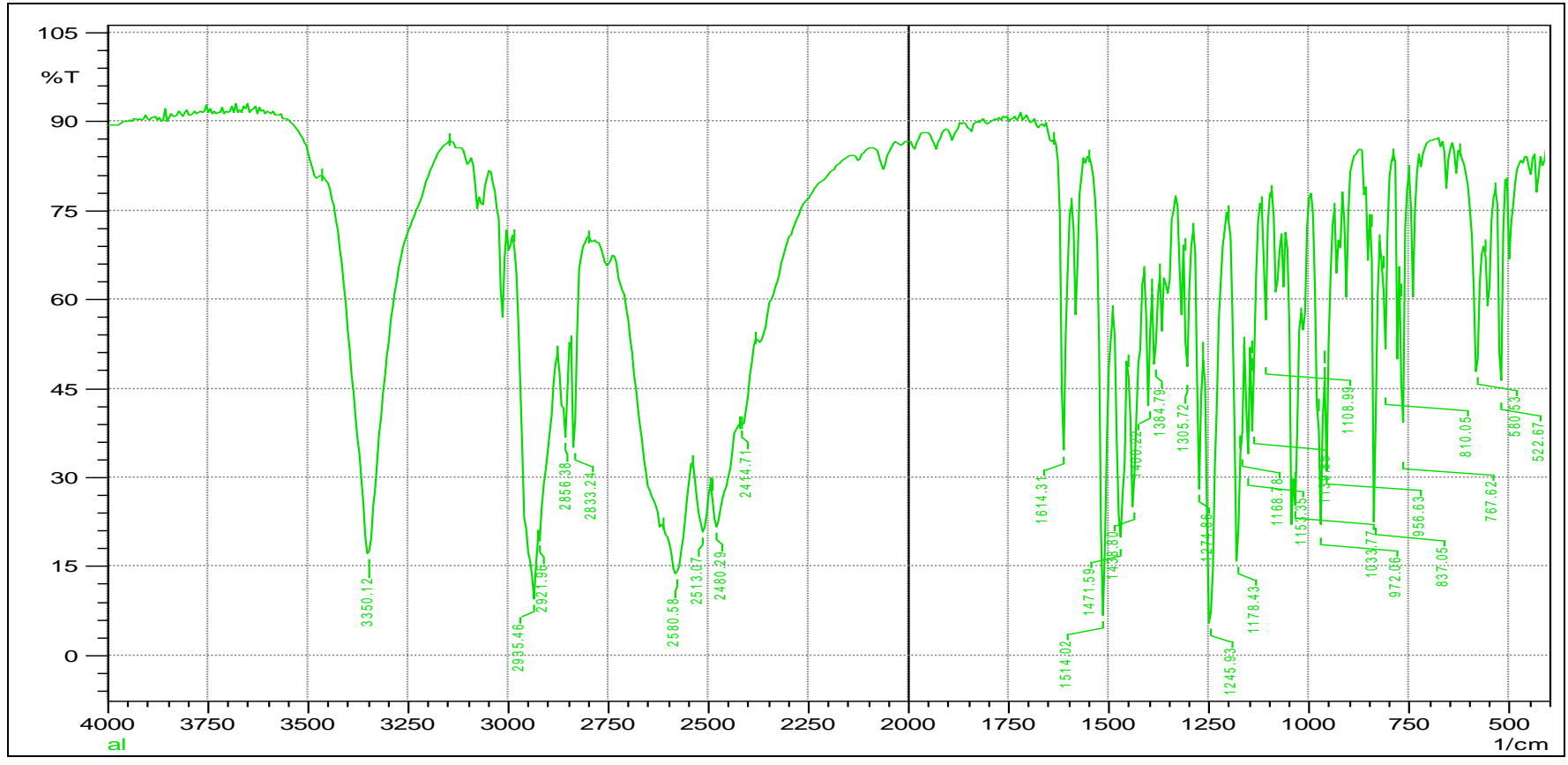

FIG. 5: FTIR OF VENLAFAXINE HYDROCHLORIDE

\section{FTIR of curve of Venlafaxine Hydrochloride and Kyron T-314}

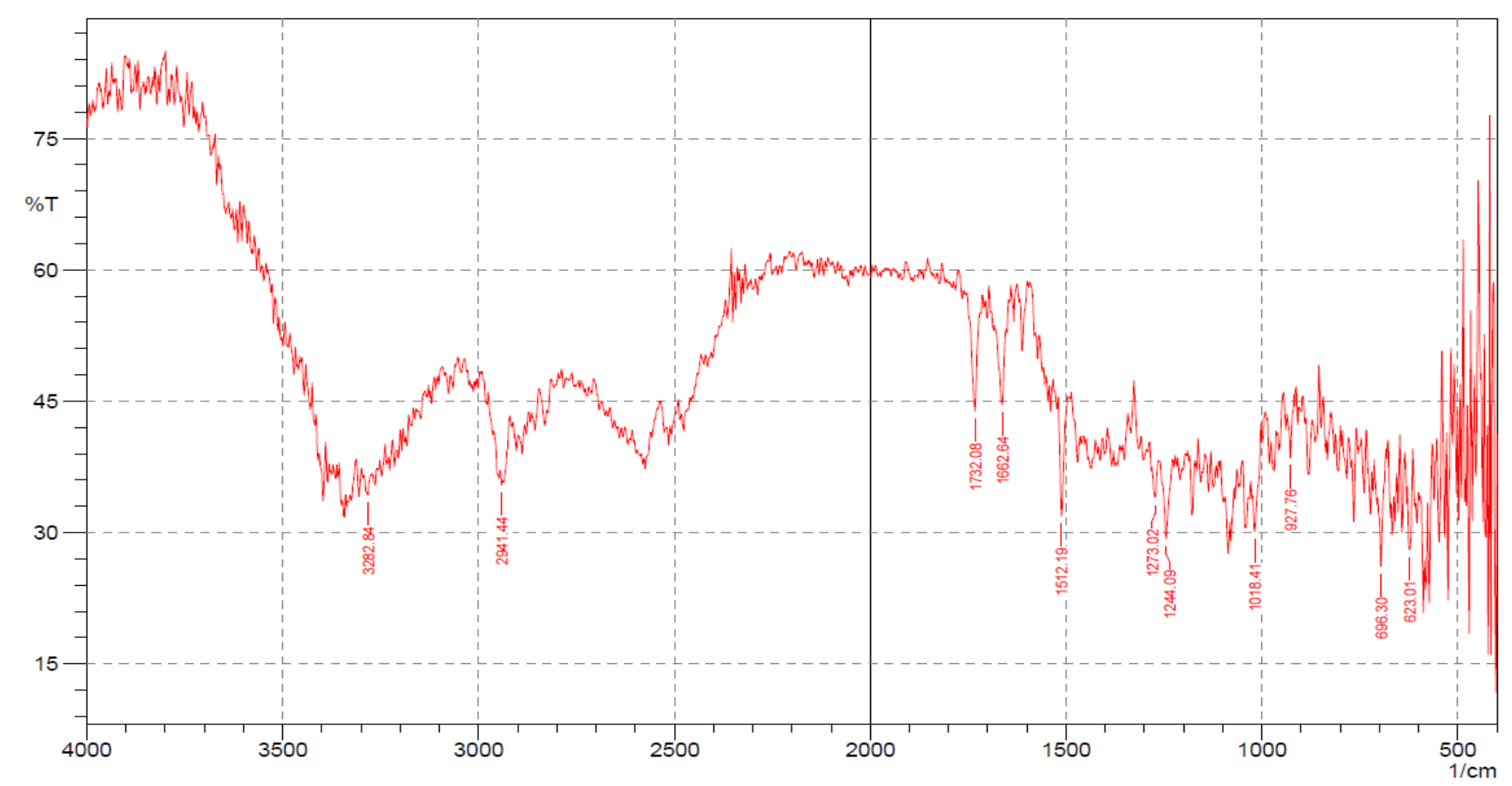

FIG. 6: FTIR OF VENLAFAXINE HYDROCHLORIDE AND KYRON T-314

The result shows that there is no incompatibility was seen in between the drug Venlafaxine hydrochloride and excipients used. All the peak of drug venlafaxine hydrochloride were present in the IR spectrum of physical mixture drug and excipients. 
Preparation of standard curve for venlafaxine hydrochloride in $0.1 \mathrm{~N} \mathrm{HCl}$ : The stock solution is used to prepare $10 \mathrm{ug} / \mathrm{ml}, 20 \mathrm{ug} / \mathrm{ml}, 30 \mathrm{ug} / \mathrm{ml}, 40 \mathrm{ug} / \mathrm{ml}$, $50 \mathrm{ug} / \mathrm{ml}, 60 \mathrm{ug} / \mathrm{ml}, 70 \mathrm{ug} / \mathrm{ml}, \quad 80 \mathrm{ug} / \mathrm{ml}, 90 \mathrm{ug} / \mathrm{ml}$, $100 \mathrm{ug} / \mathrm{ml}$ of venlafaxine in $0.1 \mathrm{~N} \mathrm{HCl}$ and analysed at $274 \mathrm{~nm}$.

TABLE 10: STANDARD CURVE OF VENLAFAXINE HYDROCHLORIDE

\begin{tabular}{ccc}
\hline Sr. No. & Concentration $(\boldsymbol{\mu g} / \mathrm{ml})$ & Absorbance $(\mathbf{n m})$ \\
\hline 1 & 0 & 0 \\
2 & 10 & 0.04 \\
3 & 20 & 0.09 \\
4 & 30 & 0.120 \\
5 & 40 & 0.167 \\
6 & 50 & 0.207 \\
7 & 60 & 0.240 \\
8 & 70 & 0.289 \\
9 & 80 & 0.329 \\
10 & 90 & 0.360 \\
11 & 100 & 0.410 \\
\hline
\end{tabular}

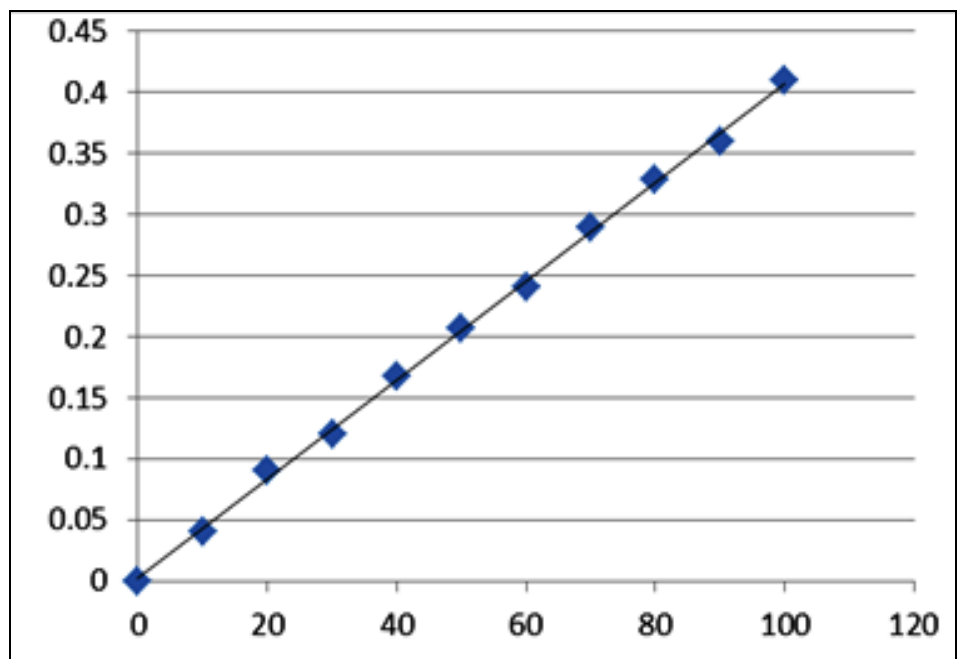

FIG. 7: STANDARD CURVE OF VENLAFAXINE HYDROCHLORIDE

Correlation coefficient $\left(R^{2}\right)=0.999$, Equation of regression line $y=0.004 x+0.002$

Where, $x=$ value for concentration, $y=$ regressed value of absorbance; $0.004=$ slope of regressed line; $0.002=y$ intercept

\section{CONCLUSIONS:}

- Sixteen Batches of Venlafaxine hydrochloride mouth dissolving tablets were prepared.

- Optimized Batch no MD-10 containing from Kyron T-314 (10\%), Microcrystalline Cellulose, Mannitol, Aspartame, Aerosil, Magnesium Stearate and Talc showed 41 second disintegration time.

- Drug content of optimized batch MD-10 was found within the limits.

- In vitro dissolution studies of MD-10 demonstrated $99.80 \%$ of drug release within 10 minutes.

\section{REFERENCE:}

1 Kuchekar B S, Atul C Badhan, Mahajan HS. Mouth dissolving tablets: a novel drug delivery system. Pharma Times 2003; 35: 7-9.

2 Virely P, Yarwood R. Zydis. A novel, fast dissolving dosage form. Manuf Chem 1998. 61: 36-37.

3 Bhushan SY, Sambhaji SP, Anant RP, Mahadik KR. New drug delivery system for elderly, Indian Drugs2003; 37 (7), 312-318.

4 Indurwade NH, Rajyaguru TH, Nakhat PD. Novel approach - fast dissolving tablets, Indian Drugs 2002; 39(8), 405-409.

5 Habib W, Khankari RK, Hontz J. Fast-dissolve drug delivery systems 2000; 61-72.

6 Dobetti L. Fast disintegrating tablets. US Patent 2003; 596,311.

7 Brown D. Orally disintegrating tablets-taste over speed. Drug Del Tech 2003; 58- 61.

8 Tanmoy Ghosh, Amitava Ghosh and Devi Prasad. A Review on New Generation Orodispersible Tablets and Its Future Prospective, Int J Pharm Sci 2011; 3, (1), 1-7.

9 Kamal Saroha, Pooja Mathur, Sureder Verma, Navneet Syan and Ajay Kumar. Mouth Dissolving Tablets: An Overviewon Future Compaction In Oral Formulation Technologies Der Pharmacia Sinica 2010; 1(1): 179-187.

10 Sahu Chandra Mohan, Chandira R. Margret. Recent Advancesin Orodispersible Tablets: A Review IJDDHR 2011;1(2), 78-83.

11 Mukesh P. Ratnaparkhi, Dr.G.P.Mohanta, Dr. Lokesh Upadhyay. Review On: Fast Dissolving Tablet 2008; 2(1),5-12.

12 Tejvir Kaur1, Bhawandeep Gill2, Sandeep Kumar3, G.D. Gupta. Mouth Dissolving Tablets: A Novel Approach to Drug Delivery, IJCPR 2011; 3(1), 1-7. 